\title{
一种基于六边形环状结构的双阻带红外频率选择表面
}

\author{
孟 真, 田昌会, 黄思宁, 范 琦, 杨百愚, 田晓霞 \\ (空军工程大学 基础部, 陕西 西安 710051)
}

\begin{abstract}
摘要: 为实现中红外大气窗口 $(3 \sim 5 \mu \mathrm{m})$ 和远红外大气窗口 $(8 \sim 14 \mu \mathrm{m})$ 的低红外透过率, 设计了 一种双频红外频率选择表面（FSS），该 FSS 由两个外侧六边形内侧圆形的环状结构组成。CST 电磁 软件仿真结果表明, 该 FSS 在中远红外两个大气窗口内的平均透过率低于 $5 \%$, 实现了中远红外的双 阻带。采用表面电流分析法分析了该 FSS 的滤波机理, 该结构通过屏内单元间的耦合形成对称电流模 式, 使散射场增强, 透过率降低, 形成了相应波段的阻带。仿真结果表明该结构具有极化稳定性, 且 对于不同入射角的 TE 波具有良好的角度稳定性, 介质层厚度和损耗角正切值对传输特性影响较小, 介电常数对其影响较大。
\end{abstract}

关键词: 中远红外; 大气窗口; 频率选择表面; 传输特性

中图分类号：TN213 文献标识码：A 文章编号：1001-8891(2020)06-0528-06

\section{Infrared Frequency Selective Surface with Dual Stopband Based on Hexagonal Ring Structure}

\author{
MENG Zhen, TIAN Changhui, HUANG Sining, FAN Qi, YANG Baiyu, TIAN Xiaoxia \\ (Department of Basic Science, Air Force Engineering University, Xi'an 710051, China)
}

\begin{abstract}
To get low transmittance in mid-infrared atmospheric windows $(3-5 \mu \mathrm{m})$ and far-infrared atmospheric windows (8-14 $\mu \mathrm{m})$, we designed a double frequency infrared frequency selective surface (FSS). This FSS is composed of two ring structures - the outer side of the structure is a hexagon and inner side is a circle. The simulation results of CST electromagnetic software show that the average transmittance of the FSS in both mid and far infrared atmospheric windows is less than 5\%; in addition, the two stopbands in infrared wavelengths are realized. The filtering mechanism of the frequency selective surface is analyzed based on the method of surface current model analysis. The structure forms a symmetrical current mode through the coupling between the unit in the screen, which enhances the scattering-field and decreases the transmission rate, forming a stopband in the corresponding band. The simulation results show that the structure has polarization stability and good angle stability for TE electromagnetic waves with different incident angles. In addition, the dielectric layer thickness and loss tangent have little effect on transmission properties, and dielectric constant has a great effect on transmission properties.
\end{abstract}

Key words: mid-far infrared, atmospheric window, frequency selective surface, transmission properties

\section{0 引言}

随着隐身技术的发展，飞机在微波波段的隐身效 果已经达到了较高的水平 ${ }^{[1]}$, 然而因飞机发动机工作、 气动摩擦均会产生热量, 形成与大气明显不同的红外 辐射, 而促使红外探测技术和红外制导武器的飞速发
展, 飞机在红外波段的隐身技术显得尤为重要 ${ }^{[2]}$ 。目 前, 飞机红外隐身大部分还是依靠涂覆低发射率材料 来实现, 所以易受材料种类和自身性能的限制 ${ }^{[3]}$ 。

频率选择表面 (frequency selective surface, FSS) 作为一种人工结构功能材料, 是一种由金属贴片单元 或是金属屏上孔径单元在加载介质上按照一定规律排

收稿日期 : 2019-07-10；修订日期 : 2019-10-29.

作者简介：孟真 (1995-)，男，山东肥城人，硕士研究生，主要从事红外辐射特性与探测技术研究。E-mail：mz2917397518@163.com。

通信作者：田昌会（1963-），男，陕西合阳人，教授，博士，主要从事红外辐射特性与探测技术研究。E-mail: tchtyb001@163.com。

基金项目：陕西省自然科学基础研究计划资助项目（2019JZ-40）。 
列而成的无限大阵列结构 ${ }^{[4-6]}$ 。FSS 对特定频率的入射 波呈现出明显的带阻或带通特性 ${ }^{[7]}$ 。在微波波段, FSS 能够降低雷达、制导舱、机舱罩的雷达散射面积 (radar cross section, RCS）; 而在红外波段, FSS 能够降低目 标在特定波段比如大气窗口内的红外辐射, 实现红 外隐身的效果 ${ }^{[8-9]}$ 。在介质表面周期性排列金属贴片 单元的贴片型 FSS 在特定的波段内具有高反射特 性, 也可以通过设计分形结构实现多波段的高反射 效果 ${ }^{[10-12]}$ 。

目前, 资料给出的结构多为复杂结构, 加工困 难 ${ }^{[13-16]}$ 。本文针对红外探测所关注的第二个和第三 个大气窗口, 即中红外和远红外大气窗口, 设计了 易于加工的基于六边形环状结构的双阻带频率选择 表面, 实现了中、远红外两个大气窗口的低透过率, 并对介质层属性的影响进行了分析。

\section{1 结构设计与分析}

为了实现在中远红外大气窗口的低透过率并减 少贴片材料的使用量, 本文设计了如图 1 所示的外侧 六边形内侧圆形的频率选择表面。如图 1(a)所示, 该 结构的中间层为介质基板，其材料为无损耗材料 $\mathrm{MgF}_{2}$, 介电常数 $\varepsilon_{\mathrm{r}}=1.9$, 两侧为具有相同周期结构且 厚度相同的金属贴片, 其材料为化学性能稳定且发射 率较低的金。图 1(a)中虚线所包围的区域为最小结构 单元, 图 1(b)为最小结构单元的正视图, 图 1(c)为最 小结构单元的侧视图。经商业仿真软件 CST ( computer simulation technology) 仿真, 对结果进行优化后, 最 终确定结构参数为 $L_{1}=0.8 \mu \mathrm{m}, L_{2}=1.5 \mu \mathrm{m}, L_{3}=1.6$ $\mu \mathrm{m}, R_{1}=0.5 \mu \mathrm{m}, R_{2}=1.1 \mu \mathrm{m}, d_{1}=1 \mu \mathrm{m}, d_{2}=0.1 \mu \mathrm{m}$ 。

\section{2 仿真与分析}

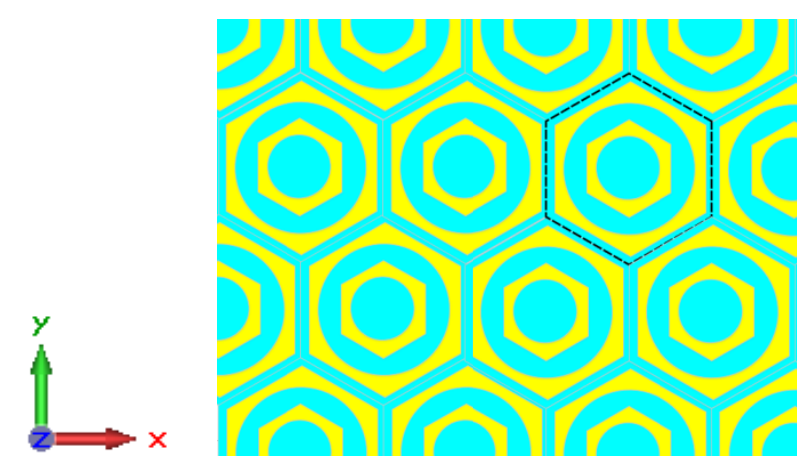

(a) FSS 结构正视图

(a) Top view of FSS

图 1 FSS 结构示意图
在商业仿真软件 CST Microwave Studio 仿真软件 中, 建立图 1 所示的结构模型, $x 、 y$ 方向上的边界条 件设置为元胞边界 (unit cell) , $\mathrm{z}$ 方向的边界条件设 置为开放边界 (open(add space))。单元结构的 $x 、 y$ 方向的周期长度均设置为 $\sqrt{3} L_{3}$, 栅格角度设置为 $60^{\circ}$ 。 采用频域求解器, 分别用 TM 波 (transverse magnetic field) 和 TE 波（transverse electric field）沿 $z$ 轴负方 向垂直入射, 对该结构进行仿真。TM 波入射时仿真 得到的光谱特性曲线如图 2 所示, 不同极化方式入射 的透过率对比曲线如图 3 所示。

由图 2 可见, 该 FSS 的透过率曲线在 3 $5 \mu \mathrm{m}$ 和 $8 \sim 14 \mu \mathrm{m}$ 两个大气窗口的平均透过率均低于 $5 \%$, 形 成了中远红外的双阻带, 并且具有陡降截止的特性。 并且在吸收率曲线中可以看到在 $6 \mu \mathrm{m}$ 附近出现了吸 收峰。由图 3 可见, $\mathrm{TM}$ 波与 $\mathrm{TE}$ 波的透过率曲线几 乎完全重合, 这说明该结构具有较强的极化稳定性。

\section{1 带阻机理分析}

当电磁波入射到 FSS 表面时, FSS 表面会激发出 表面电流, 表面电流进而产生的散射场会影响电磁波 的透过率 ${ }^{[17]}$ 。下面通过表面电流的分布情况来分析结 构在中、远红外的滤波机理。

为解释在远红外形成阻带的原因, 通过 CST 进 行仿真, 得到了 $10.62 \mu \mathrm{m}$ 处的表面电流分布情况, 如图 4 所示。图 4(a)为顶层 FSS 的表面电流分布情况, 图 4(b)为底层 FSS 的表面电流分布情况, 观察可以发 现顶层和底层的表面电流中强度较高的位置都在外 侧环上, 且顶层表面电流的强度明显高于底层。分析 出现这种现象的原因, 我们初步认为, $10.62 \mu \mathrm{m}$ 处所 对应的频点, 刚好位于外侧环谐振的中心频率, 因此 在外侧环上产生的谐振更加强烈, 进而外侧环相对内 侧环表面电流强度更高, 又因为用 CST 仿真软件进 行仿真时, 设置的电磁波入射方向为沿 $Z_{\text {min }}$ 方向, 即

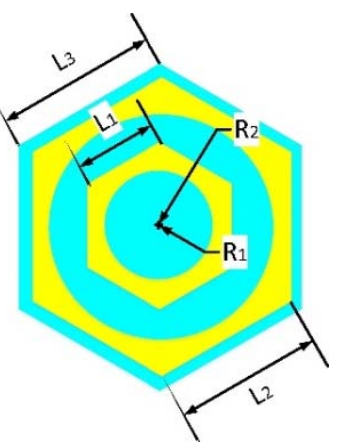

(b) 单元结构正视图

(b) Top view of unit structure

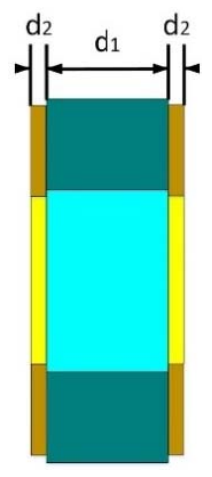

(c) 单元结构侧视图

(c) Side view of unit structure
Fig.1 Schematic diagram of FSS 


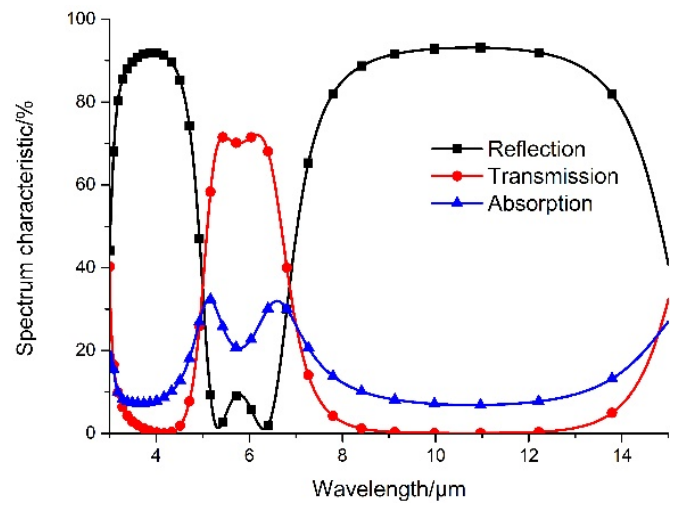

图 $2 \mathrm{TM}$ 波入射时 FSS 的光谱特性曲线

Fig. 2 The spectrum characteristic of FSS at TM wave incidence

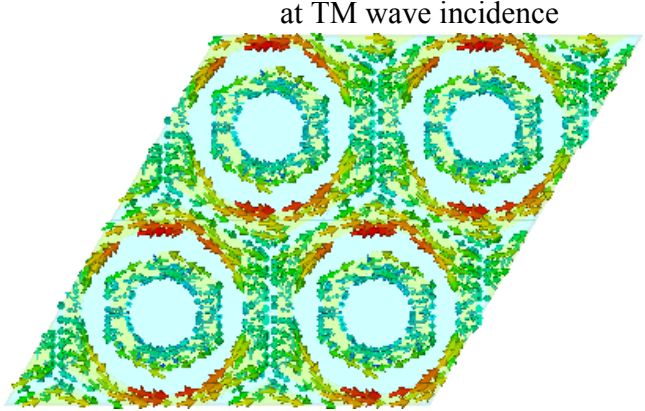

(a) 顶层 FSS 表面电流 (a) Top unit

图 $410.62 \mu \mathrm{m}$ 处表面电流分布

Fig.4

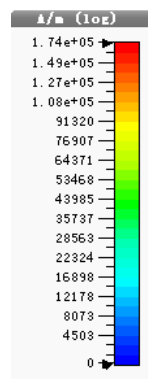

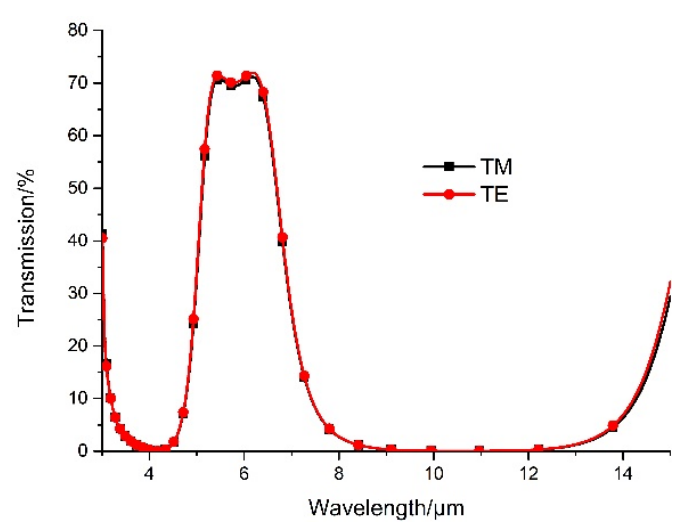

图 3 不同极化方式入射的透过率曲线

Fig. 3 The transmission when incident wave in different polarizations

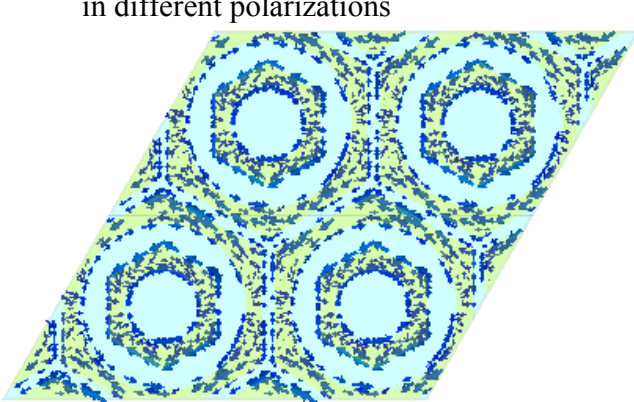

(b) 底层 FSS 表面电流 (b) Bottom unit
从 $Z_{\text {max }}$ 方向入射, 所以顶层受电磁波影响更加强烈, 进而顶层表面电流的强度高于底层。顶层和底层外侧 环上所激发的表面电流方向相反, 而每个结构单元产 生同向对称分布的表面电流, 说明这是由于屏内单元 间的耦合形成了对称电流模式, 使相关波段的透过率 降低，形成了远红外波段的阻带。

为解释在中红外形成阻带的原因, 通过 CST 进 行仿真, 得到了 $4.19 \mu \mathrm{m}$ 处的表面电流分布情况, 如 图 5 所示, 其中图 5(a)为顶层 FSS 表面电流的分布 情况, 图 5(b)为底层 FSS 表面电流的分布情况, 观 察可以发现, 该波长处内侧环状结构的表面电流强 度较高。出现这种现象的原因与 $10.62 \mu \mathrm{m}$ 处相似, $4.19 \mu \mathrm{m}$ 所对应的频点, 正好位于内侧环谐振的中心 频率附近, 因此在内侧环上产生的谐振更加强烈, 进而内侧环上的表面电流更加明显。与 $10.62 \mu \mathrm{m}$ 处 相似, 顶层的表面电流强度依然高于底层结构, 两 层表面电流的传输方向相反, 单侧表面电流形成同 向的对称分布, 同样是由于屏内单元间的耦合形成 了对称电流模式, 改变了中红外波段的透过率, 形 成了中红外波段的阻带。

总之, 中、远红外两个阻带的形成均是由于屏内 单元间的耦合形成了对称电流模式, 改变了相应波段 的透过率, 进而形成了中、远红外两个波段的阻带。
The surface current at the wavelength of $10.62 \mu \mathrm{m}$

考虑到大多数情况下电磁波并不会垂直入射到 FSS 的表面，所以对不同入射角下 FSS 的传输特性的 研究也尤为重要。图 6 为 TE 波和 TM 波以不同角度入 射到 FSS 表面时的透过率曲线, 从图 6(a)中可以看出, 当 TM 波的入射角从 $0^{\circ}$ 增加到 $60^{\circ}$ 时, $3 \sim 5 \mu \mathrm{m}$ 波段的 透过率曲线明显向长波方向移动, 且带宽明显减小, $8 \sim 14 \mu \mathrm{m}$ 的透过率曲线向短波方向有明显的移动。此 时，通过分析 TM 波入射时的吸收率曲线，如图 7(a) 所示, 发现在 3 4 $\mu \mathrm{m}$ 出现了高阶吸收, 使得在入射 角变化时 3 4 $\mu \mathrm{m}$ 的透过率出现了波动, 对中红外的 阻带效果产生了影响。如图 6(b)所示, 当 TE 波的入射 角从 $0^{\circ}$ 增加到 $60^{\circ}$ 时, FSS 的谐振点位置无较大变化, 3 $\sim \mu \mathrm{m}$ 波段的透过率曲线向长波方向有较小的移动, $8 \sim 14 \mu \mathrm{m}$ 波段的透过率曲线向短波方向有较小的移动 且带宽减小, 但整体依然保持着较低的平均透过率。 与 $\mathrm{TM}$ 波相比, $\mathrm{TE}$ 波具有较好的角度稳定性。对 $\mathrm{TE}$ 波在非大气窗口透过率降低, 且出现一个在 $6 \mu \mathrm{m}$ 附近 的透过峰的现象, 通过 CST 仿真 FSS 的反射率和吸收 率, 反射率曲线如图 7(b)所示, 且做出如下解释, 随 着入射角的增大, 该 FSS 在 5 7 $\mu \mathrm{m}$ 附近的吸收率变 化不大, 而反射率随之增大。所以当 TE 波的入射角从 $0^{\circ}$ 增加到 $60^{\circ}$ 时, $6 \mu \mathrm{m}$ 附近出现的透过峰, 是由于随 着入射角的增大, $6 \mu \mathrm{m}$ 附近的反射率不断增大导致的。

\section{2 入射角对 FSS 传输特性的影响}




\section{3 介质层属性对 FSS 传输特性的影响}

\section{1 介质层厚度对传输特性的影响}

为了研究介质层厚度对该 FSS 传输特性的影响, 分别对介质层厚度为 $0.2 \mu \mathrm{m} 、 0.5 \mu \mathrm{m} 、 0.8 \mu \mathrm{m} 、 1.2 \mu \mathrm{m}$ 和 $1.5 \mu \mathrm{m}$ 时的结构进行了仿真, 仿真结果如图 8 所示, 从图中可以看出, 介质层厚度的变化对 $3 \sim 5 \mu \mathrm{m}$ 和 $8 \sim 14 \mu \mathrm{m}$ 两个大气窗口范围内的平均透过率影响不 大, 而在 5 7 $\mu \mathrm{m}$ 范围内随着介质层厚度的增大, 透 过率有所降低。

\section{2 介质层介电常数对传输特性的影响}

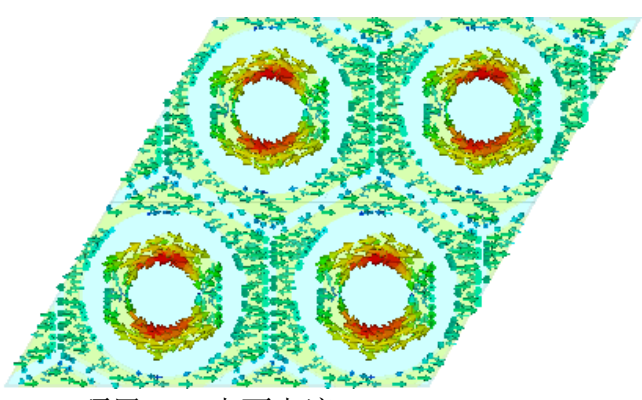

(a) 顶层 FSS 表面电流 (a) Top unit

图 $54.19 \mu \mathrm{m}$ 处表面电流分布

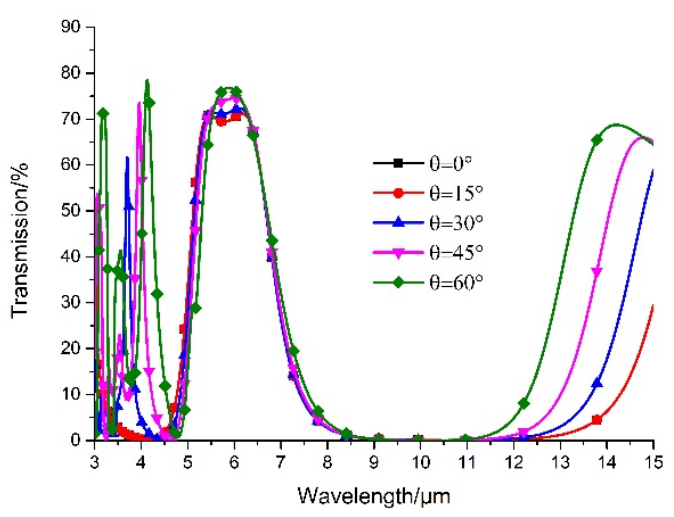

(a) TM 波 (a) TM wave

图 6 不同入射角时 FSS 的透过率曲线

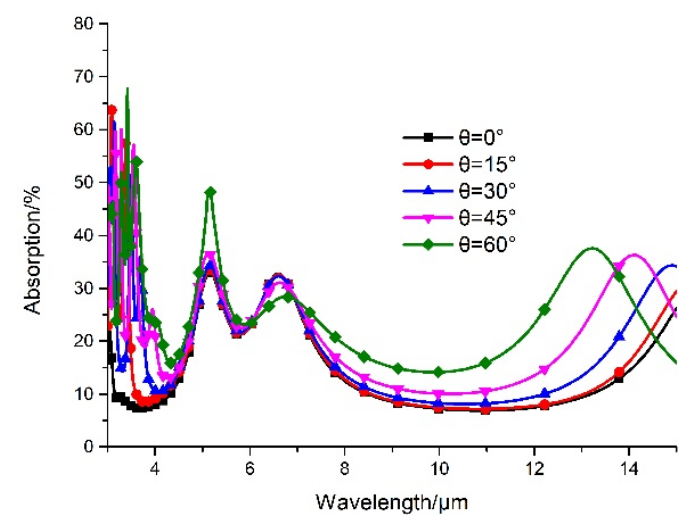

(a) 吸收率曲线 (a) Absorption curves
保持其他参数不变, 仅改变介质层的介电常数, 令 介电常数分别为 $1.9 、 2.9 、 3.9$ 和 4.9 , 用 CST 进行仿真, 仿真结果如图 9 所示。分析曲线可知, 随着介质层介电 常数的增加, 透过率曲线整体向长波方向移动, 且两个 阻带也同时向右平移, 非阻带区域透过率降低, 并且出 现了高阶吸收, 可见介质层介电常数对传输特性的影响 较大。参考相关文献, 贴片型频率选择表面可简化为串 联的 LC 振荡电路, 其谐振频率为 $f=1 / 2 \pi \sqrt{L C}$, 增大 介质层介电常数, 则相应的等效电容增大, 谐振频率降 低, 其对应的波长也就增大了 ${ }^{[18-19]}$, 所以透过率曲线向 长波方向移动, 可见仿真结果与理论分析相符合。
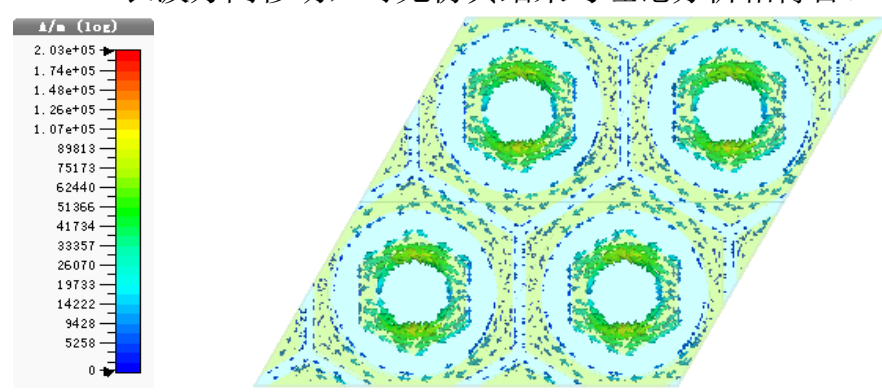

(b) 底层 FSS 表面电流 (b) Bottom unit

Fig.5 The surface current at the wavelength of $4.19 \mu \mathrm{m}$

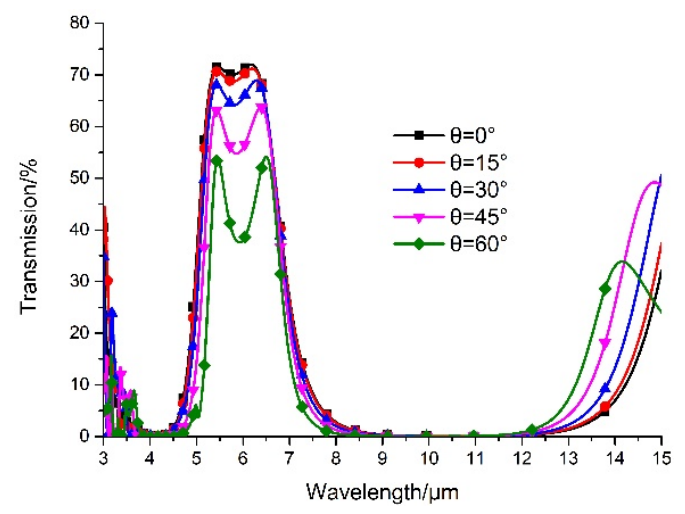

(b) TE 波 (b) TE wave

Fig.6 The transmission of FSS in different incident angle

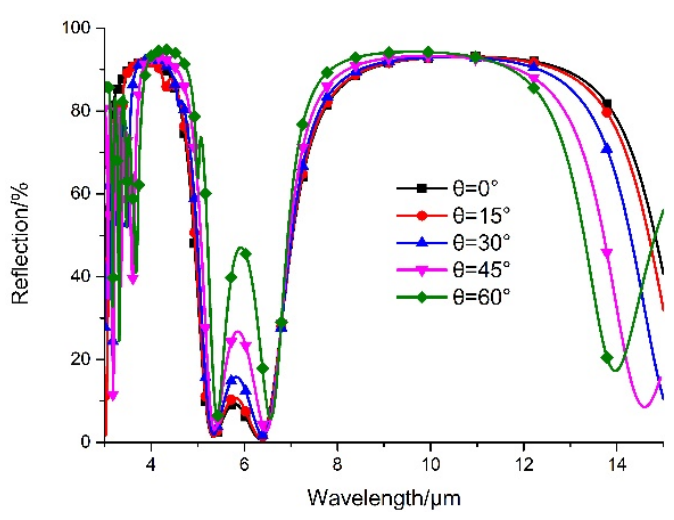

(b) 反射率曲线 (b) Reflection curves 


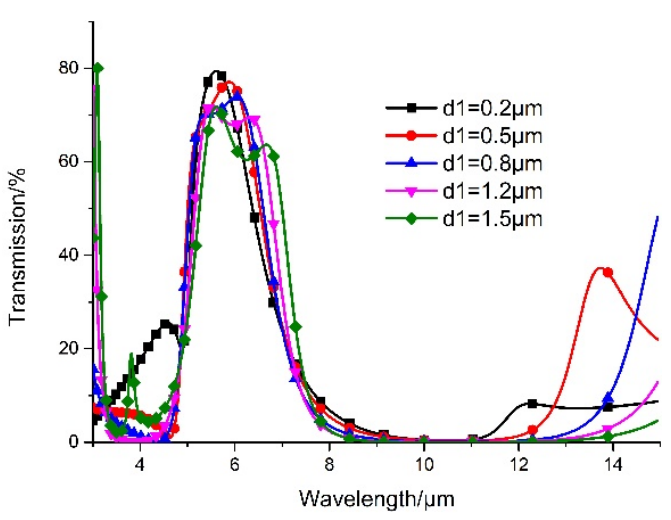

图 8 介质层不同厚度时 FSS 的透过率曲线

Fig.8 The transmission of FSS for different thickness of dielectric layer

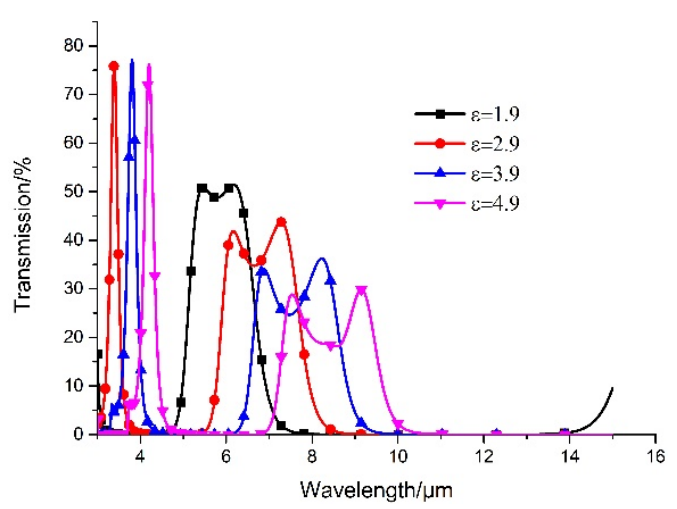

图 9 介质层不同介电常数时的 FSS 透过率

Fig.9 The transmission of FSS for different dielectric constant of dielectric

\section{3 介质层损耗角正切值对传输特性的影响}

介质损耗角正切值又称介电损耗角正切, 指的是 电解质在单位时间内每单位体积将电能转化为热能 以发热的形式而损耗的能量, 是一个用来表征电解质 对电能损耗大小的物理量。该部分通过改变介质层的 损耗角正切值, 分析了该 FSS 的传输特性, 得到的透 过率曲线如图 10 所示。分析曲线可得, 随着介质层 损耗角正切值的增大, 中远红外两个大气窗口的透过 率依然很低, 且两个阻带的位置未发生偏移, 谐振点 位置基本保持不变, 这说明介质层的损耗角正切值并 不会对阻带产生影响。但是在 $5 \sim 8 \mu \mathrm{m}$ 非工作波段的 透过率随损耗角正切值的增大而不断减小, 分析该波 段反射率和透过率后发现，非工作波段透过率的减小 是由于随损耗角正切值的增大该波段的吸收率不断 增大所导致的。所以在实际应用时, 可以选取损耗较 小的材料, 以减小对非工作波段透过率的影响。

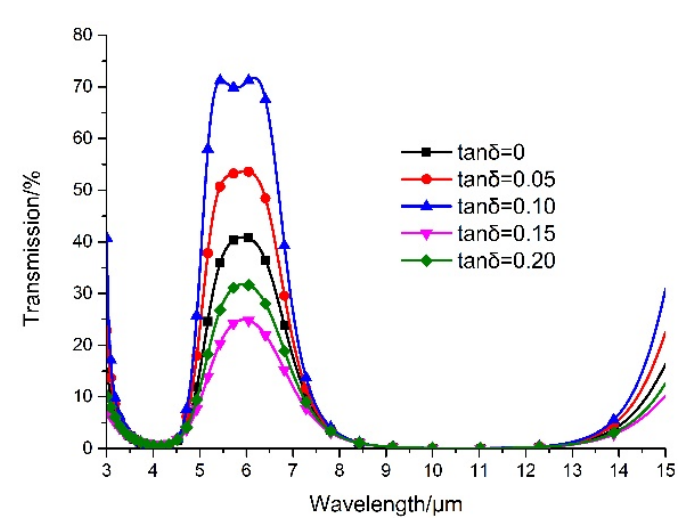

图 10 不同损耗角正切对应的 FSS 透过率

Fig.10 The transmission of FSS for different loss tangent of dielectric layer

\section{4 结论}

本文设计了一种基于外侧六边形内侧圆形的环 状结构的双阻带红外频率选择表面, 通过 CST 进行 仿真, 发现该结构实现了在中远红外即 $3 \sim 5 \mu \mathrm{m}$ 和 8 $14 \mu \mathrm{m}$ 两个工作波段的低透过率效果。该结构具 有良好的极化稳定性，使用 TM 波和 TE 波时所得到 的仿真结果相似。改变入射角度时, TE 波的仿真结 果在两个大气窗口依然能保持较低的透过率, 说明了 该结构对 TE 波具有良好的角度稳定性。进而研究了 介质层属性对该 FSS 传输特性的影响, 分别改变介质 层的厚度、介电常数和损耗角正切值, 对其进行仿真, 仿真结果表明介质层厚度和损耗角正切值对阻带位 置和带宽影响较小, 介电常数的影响较大, 随着介电 常数的增加, 透过率曲线整体向长波方向移动。

\section{参考文献:}

[1] 杨照金. 军用目标伪装隐身技术概论[J]. 应用光学, 2014, 35(3): 530. YANG Zhaojin. Introduction to camouflage stealth technology for military targets[J]. Applied Optics, 2014, 35(3): 530.

[2] 付伟. 红外隐身原理及其应用技术 [J]. 红外与激光工程, 2002, 31(1): 88-93.

FU Wei. Principle and application technology of IR stealth[J]. Infrared and Laser Engineering, 2002, 31(1): 88-93.

[3] 蒋耀庭, 王跃. 红外隐身技术与发展 [J]. 红外技术, 2003, 25(5): 7-9. JIANG Yaoting, WANG Yue. Infrared stealth technology and development[J]. Infrared Technology, 2003, 25(5): 7-9.

[4] Munk B A. Frequency Selective Surface: Theory and Design[M]. New York: Wiley, 2000.

[5] Behdad N, Mudar A J, Salehi M. A low-profile third-order bandpass frequency selective surface[J]. IEEE Transactions on Antennas and Propagation, 2009, 57(2): 460-466. 
[6] 陈晓莉, 田昌会, 王斌科, 等. 基于六边形环状结构的远红外频率选 择表面[J]. 红外技术, 2018, 40(6): 551-555.

CHEN Xiaoli, TIAN Changhui, WANG Binke, et al. A far-infrared frequency selective surface based on hexagonal loop structure[J]. Infrared Technology, 2018, 40(6): 551-555.

[7] 许志永, 张厚, 姜聿奉, 等. 一种新型双阻带频率选择表面的设计 [J]. 空军工程大学学报: 自然科学版, 2014, 15(3): 49-52.

XU Zhiyong, ZHANG Hou, JIANG Yutao, et al. A design o f frequency selective surface on a novel dual stop band[J]. Journal of Air Force Engineering University: Natural Science Edition, 2014, 15(3): 49-52.

[8] 穆金金, 王斌科, 田昌会, 等. 周期性结构对远红外辐射抑制特性分析 [J]. 激光与红外, 2016, 46(9): 1091-1095.

MU Xin, WANG Binke, TIAN Changhui, et al. Suppression analysis of periodic structure on radiation characteristics in far infrared[J]. Laser \& Infrared, 2016, 46(9): 1091-1095.

[9] 吴翔, 裴志斌, 屈绍波, 等. 基于超材料等效介质理论的带通频率选 择表面设计及验证[J]. 红外与毫米波学报, 2011, 30(5): 469-474.

WU Xiang, PEI Zhibin, QU Shaobo, et al. Design and experimental verification of band-pass frequency selective surface based on metamaterial effective medium theory[J]. Journal of Infrared and Millimeter Wave, 2011, 30(5): 469-474.

[10] Puscasu I, Schaich W L, Boreman G D. Modeling parameters for the spectral behavior of infrared frequency-selective surfaces[J]. Applied Optics, 2001, 40(1):118.

[11] Bossard J A, TANG Y, Werner D H, et al. Genetically designed multiband metallodielectric frequency selective surface filters for the mid-infrared[C]//Antennas and Propagation Society International Symposium of IEEE, 2007: 3404-3407.

[12] 王斌科, 王可欣, 田昌会, 等. 一种新型红外频率选择表面 [J]. 红外 技术, 2019, 41(1): 22-26.
WANG Binke, WANG Kexin, TIAN Changhui, et al. A novel infrared frequency selective surface[J]. Infrared Technology, 2019, 41(1): 22-26.

[13] Bossard J A, Werner D H, Mayer T S, et al. The design and fabrication of planar multiband metallodielectric frequency selective surfaces for infrared applications[J]. IEEE Transactions on Antennas \& Propagation, 2006, 54(4): 1265-1276.

[14] Shelton D J, Ginn J C, Boreman G D. Bandwidth variations in conformal infrared frequency selective surfaces[C]//IEEE Antennas and Propagation Society International Symposium, 2007: 3976-3979.

[15] Peters D W, Hadley G R, Cruzcabrera A A, et al. Infrared frequency selective surfaces for sensor applications[C]//Proceedings of SPIE, 2009, 7298: 72983L-72983L-8.

[16] Memarzadeh B, Mosallaei H. Layered plasmonic tripods: an infrared frequency selective surface nanofilter[J]. Journal of the Optical Society of America B, 2012, 29(29):2347-2351.

[17] HUANG J, WU T K, Lee S W. Tri-band frequency selective surfaces with circular ring elements[J]. IEEE Transactions on Antennas and Propagation, 1994, 42(2): 166-175.

[18] 王可欣, 王斌科, 田昌会, 等. Au/VO_2 结构可调控红外吸收器 [J]. 空军工程大学: 自然科学版, 2018, 19(5): 36-40.

WANG Kexin, WANG Binke, TIAN Changhui, et al. Tunable infrared absorber based on $\mathrm{Au} / \mathrm{VO} \_2$ structure[J]. Journal of Air Force Engineering University: Natural Science Edition, 2018, 19(5): 36-40.

[19] 郑麟, 屈绍波, 间明宝, 等. 基于 LC 耦合机制调谐设计薄屏宽带频 率选择表面[J]. 微波学报, 2016, 32(3): 1-5.

ZHENG Lin, QU Shaobo, YAN Mingbao, et al. Design broadband-pass frequency selective surface utilizing a thin substrate based on LC couplings[J]. Journal of Microwaves, 2016, 32(3): 1-5. 